\title{
Phase slips in superconducting wires with nonuniform cross section: A numerical evaluation using the string method
}

\author{
Chunyin Qiu and Tiezheng Qian* \\ Department of Mathematics, Hong Kong University of Science and Technology, Clear Water Bay, Kowloon, Hong Kong \\ Weiqing Ren \\ Courant Institute of Mathematical Sciences, New York University, 251 Mercer Street, New York, New York 10012, USA
}

(Received 19 November 2007; published 17 March 2008)

\begin{abstract}
We employ the string method to numerically evaluate the thermally activated phase slips in one-dimensional superconducting wires with nonuniform cross section. A defect (with reduced cross-sectional area) opens up a channel for phase-slip transition, for which the free-energy barrier and the preexponential factor associated with the transition rate are numerically calculated. The contribution of the defect to the total rate of transition is compared to that of the uniform section. We find that even a weak defect can greatly enhance the total rate of transition when the temperature is a bit away from $T_{c}$. In particular, for a uniform wire carrying a current that is smaller than but close to its critical current, introducing a defect can greatly lower the free-energy barrier and even destroy the metastability at all. The reduction of the critical current by defect is also discussed.
\end{abstract}

DOI: 10.1103/PhysRevB.77.104516

PACS number(s): 74.40.+k, 74.20.De, 82.20.Wt, 05.10.-a

\section{INTRODUCTION}

Suppression of superconductivity in low dimensional systems has been of theoretical and experimental interests for decades. In one-dimensional (1D) systems, i.e., wires, thermally activated phase slips are responsible for the decay of supercurrent at temperatures below the critical temperature $T_{c}$, according to a series of theoretical works by Little, ${ }^{1}$ Langer and Ambegaokar (LA), ${ }^{2}$ and McCumber and Halperin $(\mathrm{MH}){ }^{3}$ The LAMH theory ${ }^{2,3}$ provides a framework for quantitative studies of the resistance of superconducting wires below $T_{c}$. While many experimental results appear to be in good agreement with the LAMH theory, ${ }^{4,5}$ quantitative discrepancies have also been reported, ${ }^{6}$ some of which are attributed to the inhomogeneities of superconducting wires. ${ }^{7}$

The real experimental samples are inevitably inhomogeneous due to geometrical imperfection, chemical impurity, etc. Many of the previous works ${ }^{4-7}$ treat both the critical temperature $T_{c}$ and the cross-sectional area (CSA) as adjustable parameters to fit the experimental results with the LAMH predictions for ideal 1D superconducting wires of uniform CSA. However, the effects of inhomogeneities become more and more important for superconducting wires with ever reduced cross section approaching nanoscale, and a quantitative evaluation of the effects of inhomogeneities is imperative. In this paper, we will quantitatively investigate the effects of geometrical imperfection on phase-slip barrier and rate by studying 1D superconducting wires of nonuniform CSA. For simplicity, chemical impurities and size dependence of $T_{c}$ (Refs. 8 and 9) will be ignored altogether. We would like to point out that an efficient numerical scheme is needed for this purpose because it would be extremely difficult to obtain any analytical solution for wires of variable CSA.

Recently, the string method ${ }^{10-12}$ has been presented for the numerical evaluation of thermally activated rare events. This method first locates the most probable transition pathway connecting two metastable states in configuration space.
This is done by evolving strings, i.e., smooth curves with intrinsic parametrization in configuration space, into the minimal energy path (MEP). The transition rate is then computed by evaluating the height of the energy barrier and the contribution of the fluctuations around the MEP. In a previous work, ${ }^{13}$ we have shown the string method to be an efficient numerical tool for evaluating the thermally activated phase slips in uniform 1D superconducting wires. Through a comparison of the numerical results with the analytical predictions of the LAMH theory, it has been demonstrated that numerically accurate solutions can be obtained by employing the string method from the free-energy barrier to the preexponential factor (prefactor). In this paper, we investigate the effects of geometrical imperfection on the rate of thermally activated phase slips in superconducting wires of nonuniform CSA.

The paper is organized as follows. The string method is outlined in Sec. II. The model details are presented in Sec. III. The 1D superconducting wire of variable CSA is modeled by a Ginzburg-Landau (GL) free-energy functional and the corresponding time-dependent GL equation with a Langevin noise. The metastable current-carrying states, saddle points of the free-energy functional, and currentreducing phase-slip fluctuations are then qualitatively described. To investigate the competition between the phase slips induced by defects and those occurring in the uniform part of the wire, we consider nonuniform 1D wires formed by introducing a single defect into an otherwise uniform CSA. The phase slips induced by the single defect are to be numerically computed using the string method, while those occurring in the (long) uniform part can be evaluated according to the LAMH theory. The numerical results are presented in Sec. IV. Following a validation of numerical accuracy, the effects of a defect on metastable states, saddle points, freeenergy barriers, prefactors, and transition rates are numerically computed. It is shown that, in experimentally relevant temperature range, the defect-induced phase slips can even be dominant in contributing to the total transition rate be- 
cause of the lowered free-energy barrier. For this reason, the variation of free-energy barrier with defect strength (extension and depth) is further investigated for both weak and strong currents. The paper is concluded in Sec. V.

\section{STRING METHOD}

To outline the string method, we consider a system governed by the overdamped Langevin equation,

$$
\gamma \dot{q}_{i}=-\nabla_{i} V(\mathbf{q})+\zeta_{i}(t),
$$

where $\gamma$ is the damping coefficient, $\mathbf{q}$ denotes the generalized coordinates $\left\{q_{i}\right\}, \dot{q}_{i} \equiv \partial q_{i} / \partial t, \nabla_{i} \equiv \partial / \partial q_{i}$, and $\zeta_{i}(t)$ is a white noise satisfying $\left\langle\zeta_{i}(t) \zeta_{j}\left(t^{\prime}\right)\right\rangle=2 \gamma k_{B} T \delta_{i j} \delta\left(t-t^{\prime}\right)$, with $k_{B}$ denoting the Boltzmann constant and $T$ the temperature. Metastable and stable states are located in configuration space as the minima of the potential $V(\mathbf{q})$. Let $\mathbf{q}_{A}$ and $\mathbf{q}_{B}$ represent the two minima of $V(\mathbf{q})$. In terms of the topography of $V(\mathbf{q})$, the most probable fluctuation which can carry the system from $\mathbf{q}_{A}$ to $\mathbf{q}_{B}$ (or $\mathbf{q}_{B}$ to $\mathbf{q}_{A}$ ) corresponds to the lowest intervening saddle point between these two minima. The MEP is defined as a smooth curve $\widetilde{\mathbf{q}}(s)$ which connects $\mathbf{q}_{A}$ and $\mathbf{q}_{B}$ with intrinsic parametrization such as arc length $s$ and satisfies

$$
(\nabla V)^{\perp}(\widetilde{\mathbf{q}})=0,
$$

where $(\nabla V)^{\perp}$ is the component of $\nabla V$ locally normal to the path $\widetilde{\mathbf{q}}(s)$. This MEP is the most probable pathway for thermally activated transitions between $\mathbf{q}_{A}$ and $\mathbf{q}_{B}$. To numerically locate the MEP in configuration space, a string $\mathbf{q}(s)$ (a smooth curve with intrinsic parametrization by $s$ ) connecting $\mathbf{q}_{A}$ and $\mathbf{q}_{B}$ is evolved according to

$$
\dot{\mathbf{q}}=-(\nabla V)^{\perp}(\mathbf{q}) .
$$

A reparametrization is applied once in a while to enforce accurate parametrization by arc length. The stationary solution of Eq. (3) satisfies Eq. (2) which defines the MEP.

Once the MEP is determined, the lowest saddle point is known and the transition rate can be computed by evaluating the fluctuations around the MEP. ${ }^{10}$ Following Kramers' approach and its generalizations, ${ }^{14-16}$ the transition rate is given by

$$
\begin{aligned}
\Gamma(A \rightarrow & B)=\frac{\left|\lambda_{s}\right|}{2 \pi \gamma}\left[\frac{\operatorname{det} H\left(\mathbf{q}_{A}\right)}{\left|\operatorname{det} H\left(\mathbf{q}_{s}\right)\right|}\right]^{1 / 2} \\
& \times \exp \left\{-\frac{1}{k_{B} T}\left[V\left(\mathbf{q}_{s}\right)-V\left(\mathbf{q}_{A}\right)\right]\right\},
\end{aligned}
$$

where $\mathbf{q}_{s}$ is the saddle point, $H(\mathbf{q})$ denotes the Hessian matrix of $V(\mathbf{q})$, and $\lambda_{s}$ is the negative eigenvalue of $H\left(\mathbf{q}_{s}\right)$. By definition, $H\left(\mathbf{q}_{s}\right)$ has one and only one negative eigenvalue associated with the tangent vector to the MEP at the saddle point in configuration space. The determinant ratio in Eq. (4) can be numerically obtained through a stochastic simulation $^{10,12,13}$ or using a general eigensystem solver.

\section{PHASE-SLIP FLUCTUATIONS IN ONE-DIMENSIONAL SUPERCONDUCTING WIRES}

\section{A. One-dimensional superconducting wires}

For a superconducting wire below $T_{c}$, if the transverse dimension $d$ is much smaller than the temperature-dependent coherence length $\xi(T)$, then variations of the order parameter $\psi$ over the cross section of the wire are energetically prohibited. The wire can therefore be modeled as a 1D superconductor, with $\psi$ being a function of a single coordinate $x$ running along the wire. We also neglect the magnetic field assuming $d \ll \lambda(T)$, the penetration length. The GL freeenergy functional is of the form

$$
F[\psi]=\int d x\left[\frac{K}{2}\left|\partial_{x} \psi\right|^{2}-\frac{\alpha}{2}|\psi|^{2}+\frac{\beta}{4}|\psi|^{4}\right] \sigma(x),
$$

where $K=\hbar^{2} / m$, with $m$ the effective mass of a Cooper pair, $\alpha=\alpha_{0}\left(T_{c}-T\right), \alpha_{0}$ and $\beta$ are both phenomenological parameters, and $\sigma(x)$ is the $x$-dependent CSA. The time evolution of $\psi$ is governed by the time-dependent Ginzburg-Landau equation (TDGLE) with a white noise,

$$
\begin{aligned}
\gamma \frac{\partial \psi}{\partial t} & =-\frac{1}{\sigma} \frac{\delta F[\psi]}{\delta \psi^{*}}+\zeta \\
& =K \partial_{x}^{2} \psi+\alpha \psi-\beta|\psi|^{2} \psi+\frac{1}{\sigma} K \partial_{x} \sigma \partial_{x} \psi+\zeta
\end{aligned}
$$

where $\gamma$ is a damping coefficient, the asterisk denotes the complex conjugate, and $\zeta(x, t)$ is a white noise with the autocorrelation functions,

$$
\begin{gathered}
\left\langle\zeta(x, t) \zeta\left(x^{\prime}, t^{\prime}\right)\right\rangle=0, \\
\left\langle\zeta(x, t) \zeta^{*}\left(x^{\prime}, t^{\prime}\right)\right\rangle=4 \sigma^{-1} \gamma k_{B} T \delta\left(x-x^{\prime}\right) \delta\left(t-t^{\prime}\right) .
\end{gathered}
$$

This noise generates a random motion of $\psi$ in the configuration space and stabilizes the equilibrium distribution with a density proportional to $e^{-F[\psi(x)] k_{B} T}$. Compared to the case of uniform CSA, here the TDGLE involves an additional term $K \partial_{x} \sigma \partial_{x} \psi / \sigma$ arising from the spatial variation of CSA.

For computational purpose, we use the dimensionless form,

$$
\bar{F}[\bar{\psi}]=\int d \bar{x}\left[\frac{1}{2}\left|\partial_{\bar{x}} \bar{\psi}\right|^{2}-\frac{1}{2}|\bar{\psi}|^{2}+\frac{1}{4}|\bar{\psi}|^{4}\right] \bar{\sigma}(\bar{x}),
$$

for the free-energy functional. Here, the overbar denotes the dimensionless quantities, obtained with $F$ scaled by $G(T)$ $=\sigma_{o} \xi(T) \alpha^{2} / \beta$, with $\sigma_{o}$ being some characteristic CSA, $\psi$ by $\sqrt{\alpha / \beta}, x$ by the temperature-dependent correlation length $\xi(T)=\sqrt{K / \alpha}$, and $\sigma$ by $\sigma_{o}$. For wires of uniform CSA, we let $\sigma_{o}$ be the CSA itself (hence $\bar{\sigma}=1$ ), while for wire of nonuniform CSA, we let $\sigma_{o}$ be the CSA of the (long) uniform part (where $\bar{\sigma}=1$ ). Note that $\alpha^{2} / 4 \beta=H_{c}^{2}(T) / 8 \pi$ is the temperature-dependent condensation energy density, with $H_{c}(T)$ the critical field. The corresponding dimensionless TDGLE is of the form 


$$
\frac{\partial \bar{\psi}}{\partial \bar{t}}=-\frac{1}{\bar{\sigma}} \frac{\delta \bar{F}[\bar{\psi}]}{\delta \bar{\psi}^{*}}+\bar{\zeta}=\partial_{\bar{x}}^{2} \bar{\psi}+\left(1-|\bar{\psi}|^{2}\right) \bar{\psi}+\frac{1}{\bar{\sigma}} \partial_{\bar{x}} \bar{\sigma} \partial_{\bar{x}} \bar{\psi}+\bar{\zeta},
$$

in which the time is scaled by $\tau(T)=\gamma / \alpha$, i.e., $\bar{t}=t / \tau(T)$, and the dimensionless noise $\bar{\zeta}$ satisfies the autocorrelation functions,

$$
\begin{gathered}
\left\langle\bar{\zeta}(\bar{x}, \bar{t}) \bar{\zeta}^{\prime}\left(\bar{x}^{\prime}, \bar{t}^{\prime}\right)\right\rangle=0, \\
\left\langle\bar{\zeta}(\bar{x}, \bar{t}) \zeta^{*}\left(\bar{x}^{\prime}, \bar{t}^{\prime}\right)\right\rangle=4\left[k_{B} T / G(T)\right] \delta\left(\bar{x}-\bar{x}^{\prime}\right) \delta\left(\bar{t}-\bar{t}^{\prime}\right) .
\end{gathered}
$$

Through the remainder of this paper, we will mostly use the dimensionless quantities with the overbars dropped. We note that all the temperature effects are absorbed into those units in obtaining the dimensionless quantities.

\section{B. Metastable states, saddle points, and phase slips}

We adopt the ring geometry to exclude various extrinsic effects associated with the open ends. The periodic boundary condition $\psi(-l / 2)=\psi(l / 2)$ is applied to $\psi(x)$, with $x$ ranging from $-l / 2$ to $l / 2$ and $l$ the dimensionless length of the wire, and hence the accumulated change of the phase of $\psi(x)$ must be $2 n \pi$, where $n$ is an integer called the winding number. (An unambiguous determination of $n$ requires $\psi(x)$ to be nonzero everywhere.) Metastable current-carrying states corresponding to local minima of the free energy may be obtained from the stationary GL equation,

$$
\partial_{x}^{2} \psi+\left(1-|\psi|^{2}\right) \psi+\partial_{x} \sigma \partial_{x} \psi / \sigma=0 .
$$

We write $\psi(x)$ as $\psi(x)=f(x) e^{i \phi(x)}$, with $f$ and $\phi$ representing the magnitude and phase respectively. Accordingly, Eq. (9) becomes

$$
\begin{gathered}
\partial_{x}^{2} f \sigma-f\left(\partial_{x} \phi\right)^{2} \sigma+\partial_{x} f \partial_{x} \sigma+f \sigma-f^{3} \sigma=0, \\
2 \partial_{x} f \partial_{x} \phi \sigma+f \partial_{x}^{2} \phi \sigma+f \partial_{x} \phi \partial_{x} \sigma=0 .
\end{gathered}
$$

From Eq. (10b), we have $\partial_{x}\left(\sigma f^{2} \partial_{x} \phi\right)=0$, which means that in a stationary state, the supercurrent $J=\sigma f^{2} \partial_{x} \phi$ must be a constant along the wire, regardless of whether the CSA is uniform or not. In fact, in real experiments, the current is a physical quantity that is measurable and controllable. Therefore, to make physically meaningful comparisons, it is essential to keep the current invariant while introducing a defect to an otherwise uniform wire. However, here for the ring geometry adopted, the quantized winding number becomes a parameter that can be more conveniently used to calibrate the current-carrying metastable states. It is noted that for a fixed winding number, the current would be varied if a defect is introduced, especially for short wires. In this paper, we will focus on defects of weak strength, to be detailed below. Our numerical results show that for a given winding number, introducing a weak defect only leads to a negligibly small variation of current.
For uniform CSA, the metastable states are of uniform magnitude, i.e., $\partial_{x} f=0$, and the solution to Eqs. (10a) and $(10 b)$ is of the form

$$
\psi_{n}(x)=f_{n} e^{i k_{n} x}
$$

where $k_{n}=2 n \pi / l$ is the wave vector, $f_{n}=\sqrt{1-k_{n}^{2}}$ is the constant amplitude, and $n$ is the integer winding number. The (dimensionless) current density in the $\psi_{n}$ state is $j_{n}=f_{n}^{2} k_{n}=\left(1-k_{n}^{2}\right) k_{n}$. For $\psi_{n}$ to be metastable, $\left|k_{n}\right|$ must not exceed $k_{c}=1 / \sqrt{3}$, which corresponds to a critical current density $j_{c}=\sqrt{4 / 27}$.

The saddle point of the GL free energy also satisfies the stationary GL equation [Eq. (9)]. For uniform CSA, the state $\psi_{s}(x)$, defined as the saddle point intervening between two neighboring metastable states $\psi_{n}$ and $\psi_{n-1}$, was first derived by LA. ${ }^{2}$ Physically, the $\psi_{s}$ state corresponds to the most probable thermally activated fluctuation which can carry the system from $\psi_{n}$ to $\psi_{n-1}$ and vice versa. According to LAMH, to realize the transition $\psi_{n} \rightarrow \psi_{n-1}$ (or $\psi_{n-1} \rightarrow \psi_{n}$ ), the system first arrives at $\psi_{s}$ via a large fluctuation, then runs downhill in free energy toward a configuration that has a vanishing amplitude of the order parameter somewhere, and finally enters into a state with one less (more) loop in phase winding. The free-energy barrier $\Delta F$ is given by $\Delta F=F_{s}-F_{n}$ (or $\Delta F=F_{s}-F_{n-1}$ ) for the transition $\psi_{n} \rightarrow \psi_{n-1}$ (or $\psi_{n-1} \rightarrow \psi_{n}$ ), with $F_{n}$ (or $F_{n-1}$ ) and $F_{s}$ denoting the free energies of $\psi_{n}$ (or $\psi_{n-1}$ ) and $\psi_{s}$, respectively. The free-energy expression $F_{n}=-\left(1-k_{n}^{2}\right)^{2} l / 4$ shows that a lower free energy is associated with a smaller winding number (corresponding to a weaker current). Therefore, the transition $\psi_{n} \rightarrow \psi_{n-1}$ (for positive $n$ ) with a phase change of $-2 \pi$ is much more probable than the transition $\psi_{n-1} \rightarrow \psi_{n}$ with a phase change of $2 \pi$ because the free-energy barrier $\Delta F=F_{s}-F_{n}$ is much smaller. This results in a spontaneous phase-loss process responsible for the current dissipation. Based on this observation and also for the convenience of presentation, the discussions below will be focused on the transition $\psi_{n} \rightarrow \psi_{n-1}$; the extension to the transition $\psi_{n-1} \rightarrow \psi_{n}$ is straightforward. We find that the above transitional characteristic holds as well in the case of nonuniform CSA, with details given later.

Once the defect is introduced, although the physical picture of the current-reducing phase-slip fluctuations remains unchanged, analytical solutions for $\psi_{n}, \psi_{s}$, and $\Delta F$ are no longer available, and hence numerical approach becomes imperative. In Ref. 13, we have demonstrated that the numerical string method can be successfully applied to the evaluation of thermally activated phase slips in 1D wires of uniform CSA. In this paper, we will extend the string method approach to investigate the effects of geometrical imperfection (defect) on phase slips. From a numerical point of view, this extension is indeed straightforward: the metastable states $\left(\psi_{n}\right)$ can be obtained by using a standard steepest descent method, an inherent ingredient in the string method; ${ }^{10-12}$ the saddle point $\psi_{s}$ and the free-energy barrier $\Delta F$ can be evaluated from the MEP connecting $\psi_{n}$ and $\psi_{n-1}$. 


\section{Rate of transition}

The rate of transition $\Gamma$ for $\psi_{n} \rightarrow \psi_{n-1}$ can be written as

$$
\Gamma=\Omega e^{-g(\varepsilon) \Delta F},
$$

where $\Omega$ is the prefactor related to the frequency of random excursions in the configuration space and $g(\varepsilon)=G(T) / k_{B} T$ is a dimensionless energy parameter depending on the (positive) temperature parameter $\varepsilon=1-T / T_{c}$. General expression (4) for the transition rate may be directly applied to the phase-slip fluctuations. According to Eq. (4), the prefactor $\Omega$ in its dimensionless form can be formally written as

$$
\Omega=\frac{\left|\lambda_{s}^{(1)}\right|}{2 \pi}\left[\frac{\operatorname{det} H_{n}}{\left|\operatorname{det} H_{s}\right|}\right]^{1 / 2},
$$

where $H_{n}$ and $H_{s}$ are the Hessian matrices of the free-energy functional $F[\psi]$ evaluated at $\psi_{n}$ and $\psi_{s}$, respectively, and $\lambda_{s}^{(1)}$ is the lowest, negative eigenvalue of $H_{s}$. The Hessian matrix is of the form

$$
H[\psi]=\left[\begin{array}{cc}
\sigma\left(-\partial_{x}^{2}-1+3 \eta_{1}^{2}+\eta_{2}^{2}\right)-\partial_{x} \sigma \partial_{x} & 2 \sigma \eta_{1} \eta_{2} \\
2 \sigma \eta_{1} \eta_{2} & \sigma\left(-\partial_{x}^{2}-1+\eta_{1}^{2}+3 \eta_{2}^{2}\right)-\partial_{x} \sigma \partial_{x}
\end{array}\right],
$$

in which $\eta_{1}$ and $\eta_{2}$ denote the real and imaginary parts of $\psi$, respectively. Compared to the case of uniform CSA [with $\sigma(x)=1]$, here additional terms involving $\partial_{x} \sigma$ appear in the Hessian.

Extra efforts are needed because of the symmetry properties of the system. For the case of uniform CSA, the lengthdependent prefactor can be written as

$$
\Omega_{o}(l)=\frac{[2 \pi g(\varepsilon)]^{1 / 2} \Lambda l \mid \lambda_{s}^{(1)}}{(2 \pi)^{2}}\left[\frac{\operatorname{det}^{\prime} H_{n}}{\left|\operatorname{det}^{\prime \prime} H_{s}\right|}\right]^{1 / 2} .
$$

Here, $\operatorname{det}^{\prime}$ in $\operatorname{det}^{\prime} H_{n}$ indicates that the only zero eigenvalue (due to the gauge invariance of $F$ ) is to be omitted in computing the determinant, det" in $\operatorname{det}^{\prime \prime} H_{s}$ indicates that the two zero eigenvalues (one due to the gauge invariance and the other due to the translational invariance of $F$ ) are to be omitted in computing the determinant, and $\Lambda$ is a parameter arising from a standard procedure in treating the translational invariance and the associated zero eigenvalue, which can be analytically derived ${ }^{3}$ or numerically calculated. ${ }^{13}$ According to Eq. (15), the prefactor $\Omega_{o}$ for uniform CSA is proportional to the system length $l$, as required by the translational symmetry of the system: phase slips occur with equal probabilities everywhere along the wire. In fact, a complex expression for $\Omega_{o}$ has been derived by $\mathrm{MH}^{3}$ which is precise in the regime of weak-current region but is approximate in the regime of moderate and strong current. Together with LA's analytical solution for the saddle point and the free-energy barrier $\Delta F_{o}{ }^{2}$ it is therefore straightforward to obtain the transition rate.

For wires of nonuniform CAS, however, the problem becomes rather involved due to the lack of analytical solutions for $\Omega$ and $\Delta F$. The translational symmetry is now broken, and the equal probabilities for phase slips to occur along the wire do not hold anymore. Considering the additive property of the transition rates, we may formally express the total rate of transition $\Gamma_{t}$ as

$$
\Gamma_{t}=\int_{-l / 2}^{l / 2} \omega\left(x_{p}\right) e^{-g(\varepsilon) \Delta F\left(x_{p}\right)} d x_{p}
$$

where $\omega\left(x_{p}\right)$ and $\Delta F\left(x_{p}\right)$ are the prefactor per unit length and the free-energy barrier, each being a function of $x_{p}$, the center/location of the phase slip. However, Eq. (16) does not take into account the proximity effect, and hence it is valid only for slow CSA variation [with the characteristic length of CSA variation being much larger than the correlation length $\xi(T)$ ]. If the variation of CSA is relatively fast, then Eq. (16) ceases to be valid and the proximity effect has to be taken into account. This calls for a simplification of the problem that allows us to pick out the most dominant contributions to the total rate of transition.

To make the problem physical meaningful and also numerically implementable, we will work on nonuniform 1D wires formed by introducing a single defect into an otherwise uniform CSA, as illustrated in Fig. 1. The defect is mathematically of a cosine profile, i.e., $\sigma(x)=\sigma_{u}\left[1-\delta \cos \left(\pi x / l_{d}\right)\right]$ for $x \in\left(-l_{d} / 2, l_{d} / 2\right)$, inserted into a long, uniform part of $\sigma(x)=\sigma_{u}$ (whose dimensionless value is 1). Here, $\sigma_{u}$ denotes the CSA of the uniform part, $\delta$ denotes the relative defect depth, and $l_{d}$ denotes the defect length. Together, the latter two parameters $\delta$ and $l_{d}$ measure the strength of defect. Given the above defect profile, the CSA at the narrowest section is $(1-\delta) \sigma_{u}$ and the average CSA over the whole length $l$ is $\sigma_{a v}=\left(1-2 \delta l_{d} / \pi l\right) \sigma_{u}$. In the limit of weak strength $\delta l_{d} / l \rightarrow 0, \sigma_{a v} \rightarrow \sigma_{u}$, and the total rate of transition $\Gamma_{t}$ is composed of two components. The first

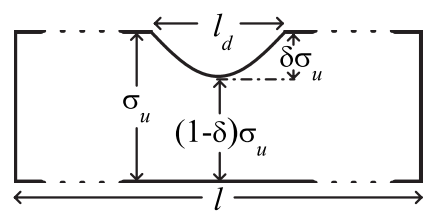

FIG. 1. A schematic illustration for a nonuniform 1D wire containing a cosinelike single defect of length $l_{d}$ and relative depth $\delta$. 
component $\Gamma_{d}$ arises from the phase slips nucleated at the defect,

$$
\Gamma_{d}=\Omega_{d} e^{-g(\varepsilon) \Delta F_{d}},
$$

and the second component $\Gamma_{u}$ arises from the phase slips in the uniform part of the wire,

$$
\Gamma_{u}=\Omega_{u} e^{-g(\varepsilon) \Delta F_{u}},
$$

where $\Omega_{d}$ and $\Omega_{u}$ denote the prefactors and $\Delta F_{d}$ and $\Delta F_{u}$ denote the free-energy barriers, with the subscripts $d$ and $u$ indicating the defect and the uniform parts, respectively. As the translational symmetry is broken by the defect, $\Omega_{d}$ is given by

$$
\Omega_{d}=\frac{\left|\lambda_{s}^{(1)}\right|}{2 \pi}\left[\frac{\operatorname{det}^{\prime} H_{n}}{\left|\operatorname{det}^{\prime} H_{s}\right|}\right]^{1 / 2},
$$

in which the only zero eigenvalue due to the gauge invariance is to be omitted in evaluating the determinants, $\operatorname{det}^{\prime} H_{n}$ and $\operatorname{det}^{\prime} H_{s}$. For defects of weak strength (i.e., of small $\delta$ and short $l_{d}$ ), the transition rate from the uniform part $\Gamma_{u}$ can be approximated by that for a wire of length $l$ and uniform CSA $\sigma_{o}=\sigma_{u}$. That is,

$$
\Gamma_{u} \cong \Gamma_{o}=\Omega_{o}(l) e^{-g(\varepsilon) \Delta F_{o}},
$$

in which $\Omega_{o}(l)$ is given by Eq. (15) and $\Delta F_{o}$ is the freeenergy barrier for uniform CSA. Equation (19) is based on the consideration that phase slips are very localized in space (of extension $\sim 1$ ) compared to the system length $l(\gg 1)$, and hence nearly all those phase slips occurring in the uniform part are not affected by the presence of a defect. (This approximation has been numerically validated: for a phase slip that occurs in the uniform part and is far away from the defect, we find that deviations from the case of uniform CSA are negligibly small from the free-energy barrier to the eigenvalues of the Hessian matrices.) Thus, the total transition rate can be written as

$$
\Gamma_{t} \cong \Gamma_{d}+\Gamma_{o} .
$$

In Sec. IV, $\Delta F_{o}, \Omega_{o}, \Delta F_{d}$, and $\Omega_{d}$ will be numerically calculated using the string method. Together, they determine the transition rate for 1D wires of nonuniform CSA. In consistency with the above, we will use the subscripts $u$ and $d$ to denote the quantities associated with the uniform part and the defect, respectively, and the subscript $o$ to denote those quantities associated with wires of uniform CSA.

\section{NUMERICAL RESULTS}

\section{A. String method applied to uniform wires: A numerical validation}

We first calculate the transition rate $\Gamma_{o}$ for wires of uniform CSA to demonstrate the numerical accuracy of the string method. For comparison with our early results, ${ }^{13}$ we consider the transition $\psi_{4} \rightarrow \psi_{3}$ for system length $l=32 \pi$. The initial winding number $n=4$ gives $k_{4}=2 n \pi / l=1 / 4$ and the current $J_{o}=k_{4}\left(1-k_{4}^{2}\right)=0.2344$, which is $61 \%$ of the critical current $J_{o c}=\sqrt{4 / 27}\left(J_{o c}=\sigma j_{o c}\right.$ with $j_{o c}=\sqrt{4 / 27}$ and $\left.\sigma=1\right)$, and hence moderately strong.
The numerical calculation is carried out as follows. The configuration $\psi(x)$ is represented by a column vector of $2 N$ entries, with the space $[-l / 2, l / 2]$ discretized by a uniform mesh of $N=400$ points. Spatial derivatives in the potential force are evaluated using central finite difference. The string is discretized by $M=101$ points in the configuration space of $\psi(x)$. The initial string is a linear interpolation between the metastable states $\psi_{4}$ and $\psi_{3}$ given by Eq. (11). The MEP is obtained by evolving the string toward the steady state according to Eq. (3), in which the potential force is now given by $-\delta F[\psi] / \delta \psi^{*}$. During the string evolution, a reparametrization by arc length is applied every ten steps.

Once the stationary MEP is obtained, the climbing image technique can be combined with the string method for the accurate calculation of saddle points. ${ }^{12,17}$ The free-energy barrier for the transition $\psi_{4} \rightarrow \psi_{3}, \Delta F_{o}=F\left[\psi_{s}\right]-F\left[\psi_{4}\right]$, is evaluated to be 0.3121 . Here, we would like to comment on the finite-size effect in relation to the numerical accuracy. The original LA expression for the free-energy barrier in the transition $\psi_{n} \rightarrow \psi_{n-1}$ is

$$
\Delta F_{o}=\frac{2 \sqrt{2\left(1-3 k_{s}^{2}\right)}}{3}-2 k_{s}\left(1-k_{s}^{2}\right) \tan ^{-1} \sqrt{\frac{1-3 k_{s}^{2}}{2 k_{s}^{2}}},
$$

in which $k_{s}$ is a wave vector associated with $\psi_{s}(x)$, given by $k_{s}=k_{n}-(2 / l) \tan ^{-1} \sqrt{\left(1-3 k_{s}^{2}\right) / 2 k_{s}^{2}}$. However, Eq. (21a) is derived in the limit of $l \rightarrow \infty$ with $k_{s} \rightarrow k_{n}$. For finite $l$, a more precise expression for $\Delta F_{o}$ can be derived based on the LA solution for $\psi_{s}$,

$$
\Delta F_{o}=\frac{2 \sqrt{2\left(1-3 k_{s}^{2}\right)}}{3}+\frac{l}{4}\left(k_{s}^{2}-k_{n}^{2}\right)\left(2-k_{n}^{2}-k_{s}^{2}\right) .
$$

Substituting the numerically evaluated $k_{s}$ into Eq. (21a) yields $\Delta F_{o}=0.3385$. If Eq. (21b) is used instead, then $\Delta F_{o}$ $=0.3130$. We note that the numerical value 0.3121 obtained using the string method shows a very small relative deviation $(\approx 0.3 \%$, which can be further reduced by using finer grids) from the theoretical value of 0.3130 . For comparison purpose, we also note that Tarlie et al. ${ }^{18}$ have shown the relative shift due to the finite $l$ to be of order $1 / l$ and given the leading-order term of correction, which yields $\Delta F_{o}=0.3119$ in the present case.

As to the prefactor $\Omega_{o}$, the discretized form of the Hessian matrices is used for numerical calculation. The determinant ratio in Eq. (15) for $\Omega_{o}$ is directly obtained using a general eigensystem solver, instead of the numerical technique presented in Ref. 13. We obtain $\lambda_{\mathrm{s}}^{(1)}=-0.3759, \Lambda=0.8528$, and the determinant ratio $\operatorname{det}^{\prime} H_{4} /\left|\operatorname{det}^{\prime \prime} H_{s}\right|=1.256$, and hence the prefactor $\Omega_{o}=0.9149 \times[2 \pi g(\varepsilon)]^{1 / 2}$, which is in reasonable agreement with $0.7326 \times[2 \pi g(\varepsilon)]^{1 / 2}$ from MH's approximate expression. These results also represent a numerical improvement (by 4\%-16\%) over those obtained in Ref. 13 because (i) the climbing image technique has been implemented for accurate calculation of saddle points, and (ii) the determinant ratio is directly evaluated by employing a general eigensystem solver, which does not involve statistical 
fluctuations and slow convergence incurred by the stochastic-simulation method used in Ref. 13.

\section{B. Defect-induced phase slips}

Now, we turn to the rate of defect-induced phase slips $\Gamma_{d}$ for nonuniform wires containing a single defect, as illustrated in Fig. 1. Here, for easy comparison, we study the transition $\psi_{4} \rightarrow \psi_{3}$ using the same system length $l=32 \pi$. The defect is of the cosine profile defined in Sec. III C, with the defect length given by $l_{d}=\pi$ and defect depths given by $\delta$ $=0.1$ and $\delta=0.3$. The metastable states are readily obtained by the steepest descent method starting from the initial states described by Eq. (11). The condition of constant current along the ring $\left(J=\sigma f^{2} \partial_{x} \phi=\right.$ const $)$ for all stationary states is numerically verified. As stated earlier, in this paper, we only consider defects of weak strength to ensure that for a fixed winding number, the current variation is negligibly small once a defect is introduced. This weak-defect assumption has been checked. For example, in the $\psi_{4}$ state, the current is found to be $J_{d}=0.2337$ for $\delta=0.1$ and $J_{d}=0.2323$ for $\delta=0.3$, which deviate from the current for no defect (the uniform case) $J_{o}=0.2344$ by $0.21 \%$ and $0.81 \%$, respectively. Note that the free-energy barrier associated with defect-induced phase slips depends on the current and the defect strength (i.e., length and depth). Our purpose is to investigate how the free-energy barrier varies with the defect strength, with the current kept invariant. In numerical computation, it is easy to fix the winding number in the presence of a defect. However, for a fixed winding number, the current has to change with the defect strength. In this paper, we focus on the weak defects, for which it has been verified that for a given winding number, introducing a defect only leads to negligibly small current variation. Therefore, by fixing the winding number, we can measure the defect-induced change of free-energy barrier for a nearly invariant current.

To implement the string method, an initial string is prepared so that while evolving according to Eq. (3), it is driven toward a stationary solution, i.e., a MEP, in which the saddle point $\psi_{s}(x)$ corresponds to a phase slip centered at the defect center $x=0$. The MEPs connecting the current-carrying states $\psi_{4}$ and $\psi_{3}$ are shown in Fig. 2 for the three cases of $\delta=0$ (no defect), 0.1 , and 0.3 . The free-energy barrier associated with the defect-induced phase slip is found to be $\Delta F_{d}=0.2534$ for $\delta=0.1$ and $\Delta F_{d}=0.1392$ for $\delta=0.3$, respectively. Together with $\Delta F_{o}=0.3121$ for $\delta=0$, they show the anticipated tendency that $\Delta F_{d}$ decreases with increasing defect depth $\delta$.

It is physically interesting to look into the defect-induced free-energy variations of the metastable states and saddle points. As shown in Fig. 2, the metastable states acquire an increasing free energy as the defect is introduced and deepened, but the saddle points display the opposite trend. These may be heuristically understood as follows. For wires of uniform CSA, the metastable states described by Eq. (11) have constant magnitude $|\psi|=f_{n}$ and phase gradient (wave vector) $\partial_{x} \phi=k_{n}$. Once a defect of reduced CSA is introduced, the magnitude $|\psi|$ becomes locally suppressed (with $\partial_{x} \phi$ locally enhanced in the defect segment to maintain a constant current along the wire), as shown in Fig. 3(a). Physically, the

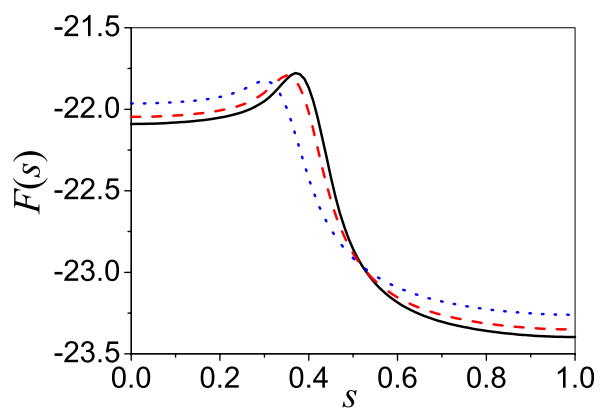

FIG. 2. (Color online) Dimensionless free energy $F$ along the MEP from $\psi_{4}$ to $\psi_{3}$, plotted as a function of the arc length $s$ in the $\psi(x)$-function space, for $\delta=0$ (solid line), 0.1 (dashed line), and 0.3 (dotted line), with $l=32 \pi$ and $l_{d}=\pi$. The $\psi_{4}$ state is taken as the reference point where $s=0$ and the arc length measured along the MEP is normalized by that from $\psi_{4}$ to $\psi_{3}$, hence $s$ runs from 0 to 1 .

locally reduced CSA produces a weak point in the superconducting wire, making the condensation locally less complete in metastable states compared to the case of no defect, and hence results in a higher free energy. The opposite trend is displayed by the saddle points which are characterized by a local order-parameter variation (suppression) toward the normal state, as shown in Fig. 3(b). A weak point would only make such variation more likely to occur, and hence it results in a lower free energy. The suppressed order-parameter magnitude and the enhanced phase gradient in metastable states would make phase slips prefer to occur at the defect, as exhibited by the reduced free-energy barrier and also the
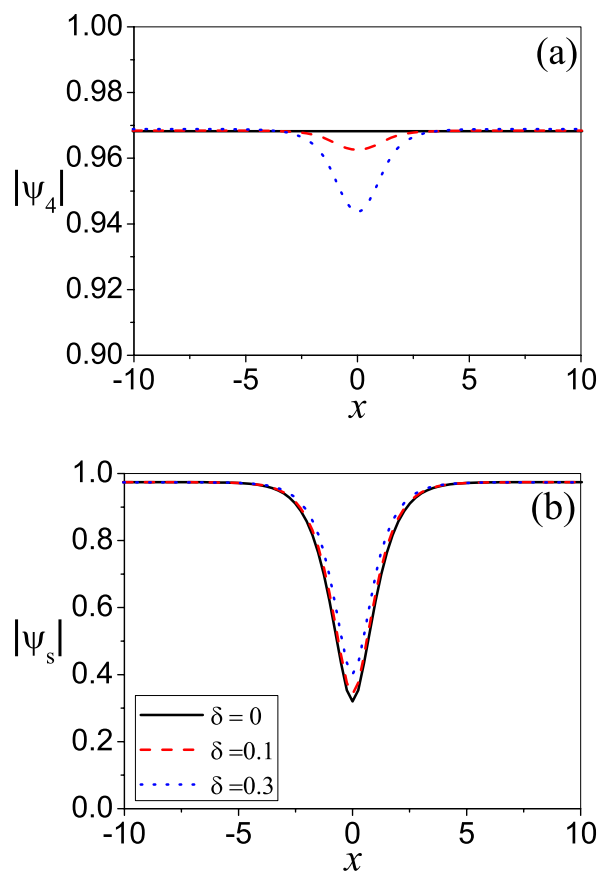

FIG. 3. (Color online) The variations of the order-parameter magnitude in the initial metastable state $\left[\left|\psi_{4}(x)\right|\right.$ in (a)] and the saddle point $\left[\left|\psi_{s}(x)\right|\right.$ in (b)], evaluated for $\delta=0$ (solid line), 0.1 (dashed line), and 0.3 (dotted line), with $l=32 \pi$ and $l_{d}=\pi$. For clear illustration, only the segments of spatially varying $|\psi(x)|$ are shown. 


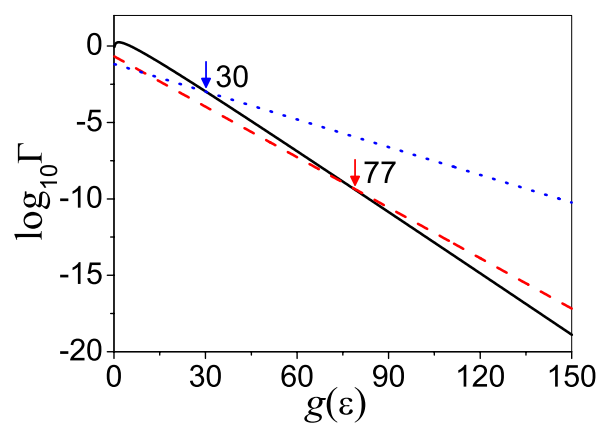

FIG. 4. (Color online) The variations of the transition rates $\Gamma_{o}$ (solid line) and $\Gamma_{d}$ (dashed and dotted lines for $\delta=0.1$ and 0.3 , respectively) with $g(\varepsilon)$, evaluated for $l=32 \pi$ and $l_{d}=\pi$.

reduced arc length along the MEP from the metastable state to the saddle point.

As to the prefactor $\Omega_{d}$ associated with the phase slips confined to the defect, we obtain $\Omega_{d}=0.2141$ for $\delta=0.1$ and $\Omega_{d}=0.0669$ for $\delta=0.3$, respectively, using Eq. (18). We note that the prefactor associated with the phase slips in the uniform part is given by $\Omega_{u} \cong \Omega_{o}=0.9149[2 \pi g(\varepsilon)]^{1 / 2}$ (in Sec. IV A), which depends on the dimensionless energy scale $g(\varepsilon)=G(T) / k_{B} T$. From the physical criterion for the applicability of the rate theory, $g(\varepsilon) \Delta F \gg 1$, and the fact that for uniform CSA the highest free-energy barrier ${ }^{2}$ is $\Delta F=2 \sqrt{2} / 3 \cong 1$ in the weak-current limit of $\left|k_{n}\right| \rightarrow 0$ (and hence the current density $j_{n}=\left(1-k_{n}^{2}\right) k_{n} \rightarrow 0$ in $\left.\psi_{n}\right)$, we deduce $g(\varepsilon) \gg 1$ and thus $\Omega_{d} \ll \Omega_{o}$. Therefore, compared to those occurring in the uniform part of the wire, the phase slips confined to the defect would occur with a rate that is enhanced by a lowered free-energy barrier yet suppressed by a reduced prefactor. Note that $\Omega_{o}$ contributed by the uniform part is proportional to the total length $l\left(l \gg l_{d}\right)$ according to Eqs. (15) and (19). Therefore, for a very long wire with many defects, a comparison of $\Omega_{d}$ and $\Omega_{o}$ is meaningful only if the density of defects is given. That is, $\Omega_{d}$ for a single defect is to be compared to $\Omega_{o}$ for $l$ equaling the average separation of two neighboring defects or the average length of the uniform segment in between. Note that the interaction between the defects is negligible if they are well separated, i.e., $l \gg 1$.

The relative importance of the defect-induced transition rate $\Gamma_{d}$ depends on the temperature-dependent energy parameter $g(\varepsilon)$. Figure 4 shows the variations of the transition rates $\Gamma_{o}$ and $\Gamma_{d}$ with $g(\varepsilon)$. Given the parameters above, we find that $\Gamma_{d} \geq \Gamma_{o}$ is obtained for $g(\varepsilon) \geq 77$ at $\delta=0.1$ and $g(\varepsilon) \geq 30$ at $\delta=0.3$. That is, for sufficiently large $g(\varepsilon)$, the defect-induced transition rate may overpass that contributed by the uniform part, with $\Gamma_{d}$ dominant in the total transition rate $\Gamma_{t}=\Gamma_{d}+\Gamma_{o}$. The reason is that with $\Gamma_{d} \sim e^{-g(\varepsilon) \Delta F_{d}}$, $\Gamma_{o} \sim e^{-g(\varepsilon) \Delta F_{o}}$, and $\Delta F_{d}<\Delta F_{o}$, a sufficiently large $g(\varepsilon)$ would let $\Gamma_{d}$ win out irrespective of the prefactors. Below but close to $T_{c}$, we have the critical field $H_{c}(T) \cong 1.73 \varepsilon H_{c 0}$ and correlation length $\xi(T) \cong 0.74 \varepsilon^{-1 / 2} \xi_{0}$, with the numerical coefficients determined from the BCS theory, ${ }^{19}$ and hence $g(\varepsilon)=\sigma_{u} \xi H_{c}^{2} / 2 \pi k_{B} T \cong \varepsilon^{3 / 2} g_{0}$, where $H_{c 0}$ and $\xi_{0}$ are the zerotemperature critical field and coherence length, and $g_{0}=0.352 \sigma_{u} \xi_{0} H_{c 0}^{2} / k_{B} T_{c}$ which is dependent on the CSA of the uniform part $\sigma_{u}$ and the material properties of superconductor. Note that the dimensionless parameter $g(\varepsilon) \propto \varepsilon^{3 / 2}$ rapidly increases as the temperature is lowered away from $T_{c}$. Here, we want to emphasize that in generating Fig. 4, a fixed dimensionless defect length $\left(l_{d}=\pi\right)$ has been used. However, $l_{d}$ is defined to be the real defect length scaled by the temperature-dependent correlation length $\xi(T)$. Therefore, Fig. 4 does not correspond to a real defect [whose dimensionless length varies as $1 / \xi(T)]$. While $g(\varepsilon)$ varies with the temperature $T$, we also let the defect length change with $T$ in order to keep the dimensionless length $l_{d}$ constant. Note that the purpose of this paper is to provide a numerical study of the defect-induced phase slips. Therefore, we have chosen to present our results in terms of various dimensionless quantities, controlled by the dimensionless parameters defined in the GL model. For a real defect, the temperature dependence comes from those dimensionless parameters and units that vary with $T$, e.g., $l_{d} \propto\left(T_{c}-T\right)^{1 / 2}, g(\varepsilon) \propto\left(T_{c}-T\right)^{3 / 2}$, and $\tau \propto\left(T_{c}-T\right)^{-1}$.

To show the relevance of the temperature-dependent parameter $g(\varepsilon)$, we consider the real 1D tin (Sn) superconducting wires (whiskers) extensively used in the measurement of current dissipation. ${ }^{4-7}$ Using $T_{c} \sim 3.7 \mathrm{~K}, \quad \xi_{0} \sim 0.298 \mu \mathrm{m}$, and $H_{c 0} \sim 306 \mathrm{Oe}$ provided in Ref. 5 and setting $\sigma_{u} \sim 2.0 \times 10^{-9} \mathrm{~cm}^{2}$ for the CSA of the uniform part, we have $g_{0} \sim 3.83 \times 10^{6}$. For $\delta=0.1, \quad \Gamma_{d}$ crosses $\Gamma_{o}$ at $g(\varepsilon)=77$. Substituting this value into $g(\varepsilon) \cong \varepsilon^{3 / 2} g_{0}$ yields $\varepsilon=7.41 \times 10^{-4}$, which corresponds to $\Delta T=2.74 \mathrm{mK}$ and $\xi(T) \cong 0.74 \varepsilon^{-1 / 2} \xi_{0}=8.10 \mu \mathrm{m}$. Here, $\Delta T=T_{c}-T$ measures the deviation of $T$ (at which $\Gamma_{d}=\Gamma_{o}$ ) from $T_{c}$. Accordingly, the dimensionless parameters $l_{d}=\pi$ and $l=32 \pi$ used in Fig. 4 correspond to defect length $l_{d} \xi=25.45 \mu \mathrm{m}$ and system length $l \xi=814.3 \mu \mathrm{m}$. Similarly, for $\delta=0.3, \Gamma_{d}$ crosses $\Gamma_{o}$ at $g(\varepsilon)=30$, corresponding to $\varepsilon=3.95 \times 10^{-4}, \Delta T=1.46 \mathrm{mK}$, and $\xi=11.1 \mu \mathrm{m}$. Accordingly, the dimensionless parameters $l_{d}=\pi$ and $l=32 \pi$ correspond to defect length $34.86 \mu \mathrm{m}$ and system length $1115.9 \mu \mathrm{m}$. Here, we note that the values of $\Delta T$ obtained for $\delta=0.1$ and 0.3 are close to the boundary of the experimental temperature range for the overall rate to be observable. ${ }^{5}$ Therefore, in order to have dominant defectinduced phase slips in the experimental temperature range for measurable overall rates, constrictions severer than $l_{d}=\pi$ and $\delta=0.3$ are needed to further reduce $\Delta T$. This fact will be made evident by the numerical results in Sec. IV C. Finally, we want to point out that the above estimation has been made by using the GL free-energy functional with variable CSA and taking into account the proximity effect. It will be shown in Sec. IV C that the LAMH prediction for the defect-induced energy barrier, locally determined by the CSA at the narrowest section $(1-\delta) \sigma_{u}$, is always an underestimate, corresponding to the saturation value in the limit of $l_{d} \rightarrow \infty$, which requires the defect to be much longer than the correlation length $\xi(T)$.

\section{More on defect-induced energy barriers}

The numerical results obtained above show that, at temperatures appreciably below $T_{c}$, the defect-induced transition rate $\Gamma_{d}$ dominates in the total transition rate $\Gamma_{t}$ because of the 

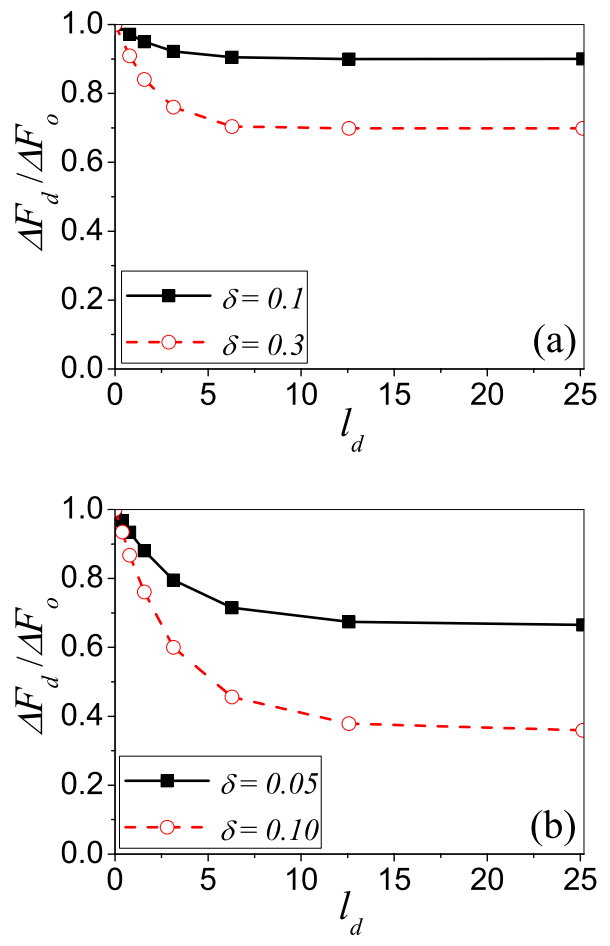

FIG. 5. (Color online) The normalized free-energy barrier $\Delta F_{d} / \Delta F_{o}$ plotted as a function of the defect length $l_{d}$ for different values of defect depth $\delta$. (a) The case of weak current with $n=1$ and $J_{d} \cong 0.041 J_{o c}$. (b) The case of strong current with $n=24$ and $J_{d} \cong 0.837 J_{o c}$. Here, the results are obtained for $l=128 \pi$, with $\Delta F_{o}=0.905$ at $n=1, \Delta F_{o}=0.099$ at $n=24$, and $J_{o c}=\sqrt{4 / 27}$ being quantities associated with a uniform wire of CSA $\sigma_{o}=\sigma_{u}$.

reduced energy barrier. Below, we will further investigate how the defect strength affects the free-energy barrier $\Delta F_{d}$ for both weak and strong currents. For fixed system length $l=128 \pi$, Fig. 5 shows the variations of the normalized freeenergy barrier $\Delta F_{d} / \Delta F_{o}$ with the defect length $l_{d}$ for different values of defect depth $\delta$. Figure 5(a) is for a weak current with $n=1$ and $J_{d} \cong 0.041 J_{o c}$, while Fig. $5(\mathrm{~b})$ is for a strong current with $n=24$ and $J_{d} \cong 0.837 J_{o c}$. Here, $\Delta F_{o}$ is the freeenergy barrier evaluated for a uniform wire of CSA $\sigma_{o}=\sigma_{u}$ using the same winding number $n(=1$ or 24$)$ and system length $l$, and $J_{o c}=\sqrt{4 / 27}$ is the critical current for this uniform wire.

Figures 5(a) and 5(b) show that the free-energy barrier $\Delta F_{d}$ decreases with increasing defect strength (either $l_{d}$ or $\delta$ ). The spatial correlation of the order parameter makes the defect effectively "invisible" as $l_{d} \rightarrow 0$ [compared to $\xi(T)$ ] and thus $\Delta F_{d} / \Delta F_{o} \rightarrow 1$ in that limit. The defect takes effect when $l_{d}$ approaches 1 , the characteristic extension of a phase-slip event. When $l_{d}$ sufficiently becomes large, $\Delta F_{d}$ reaches a saturation value corresponding to the strongest suppression for a given defect depth $\delta$. In particular, for weak current, the saturation value of $\Delta F_{d}$ can be approximated by the freeenergy barrier associated with a uniform wire of CSA $\sigma_{u}(1-\delta)$, which is the cross-sectional area at the narrowest point of the defect. In Fig. 5(a), the saturation values of the normalized free-energy barrier $\Delta F_{d} / \Delta F_{o}$ are found to be 0.901 and 0.699 for $\delta=0.1$ and 0.3 , respectively, well ap-

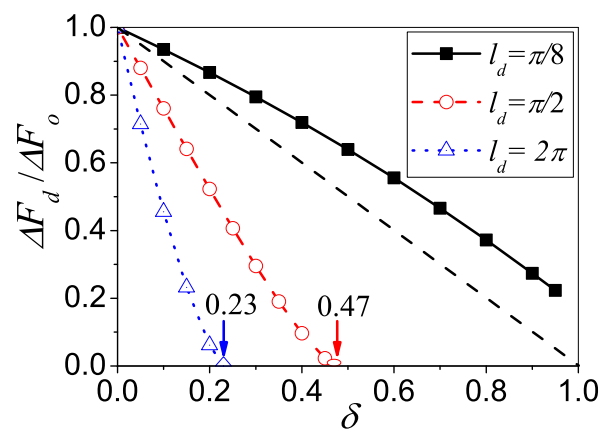

FIG. 6. (Color online) The normalized free-energy barrier $\Delta F_{d} / \Delta F_{o}$ plotted as a function of defect depth $\delta$ for different values of defect length $l_{d}$. Same as before, $\Delta F_{o}=0.099$ is the free-energy barrier associated with the uniform wire of CSA $\sigma_{o}=\sigma_{u}$ for $n=24$ and $l=128 \pi$ [the strong-current case in Fig. 5(b)]. The arrows denote the critical defect depth $\delta_{c}$ at which $\Delta F_{d} / \Delta F_{o}$ vanishes, and the dashed line is for $\Delta F_{d} / \Delta F_{o}=1-\delta$.

proximated by $1-\delta$. (Noted that $\Delta F_{o}$ used in normalization is for the uniform wire of CSA $\sigma_{o}=\sigma_{u}$. ) This simple approximation has been intuitively mentioned in some experimental studies. It fails, however, for strong currents. As shown in Fig. 5(b), the saturation values of $\Delta F_{d} / \Delta F_{o}$ are 0.665 and 0.359 for $\delta=0.05$ and $\delta=0.1$, respectively, well below the corresponding values of $1-\delta$. This fast suppression of the free-energy barrier is due to the locally enhanced current density at the narrowest section. In addition, the saturation value of the free-energy barrier at $l_{d} \rightarrow \infty$ corresponds to the LAMH prediction that can be made using the CSA and the current density at the narrowest section.

For strong currents, the free-energy barrier $\Delta F_{d}$ is severely suppressed and eventually vanishes as $\delta$ reaches a critical value. This trend is already indicated by Fig. 5(b) where a fast decrease of $\Delta F_{d} / \Delta F_{o}$ with increasing $\delta$ is seen. To show this behavior more clearly, we plot the normalized free-energy barrier $\Delta F_{d} / \Delta F_{o}$ as a function of defect depth $\delta$ for different values of defect length $l_{d}$, with $n=24$ and $l=128 \pi$ fixed [the strong-current case in Fig. 5(b)]. As shown in Fig. $6, \Delta F_{d} / \Delta F_{o}$ decreases with increasing $\delta$ and the slope of such decrease increases with increasing defect length $l_{d}$, in agreement with Fig. 5(b). Once again, we see $\Delta F_{d} / \Delta F_{o}<1-\delta$ for (relatively) long defects, such as $l_{d}=\pi / 2$ and $2 \pi$. For $l_{d}=2 \pi, \Delta F_{d}$ rapidly decreases with increasing $\delta$ and hits zero at a critical value of $\delta, \delta_{c}=0.23$. This is the point at which the current-carrying state of the winding number $n=24$ loses its metastability. It is interesting to look at the variation of the current density along the wire when $\delta=\delta_{c}$. For $l_{d}=2 \pi$, the critical value of defect depth is $\delta_{c}=0.23$, at which the current density in the uniform part is found to be $j_{u}=0.3204$, below the critical value for a wire of uniform CSA, $j_{o c}=\sqrt{4 / 27} \approx 0.3849$, but the current density at the narrowest point of the defect is $j_{\max }$ $=j_{u} /\left(1-\delta_{c}\right)=0.4161$ (with the current $J_{d}=\sigma j$ being a constant), which is slightly above $j_{o c}$. For a shorter defect with $l_{d}=\pi / 2$, the critical value of defect depth is $\delta_{c}=0.47$, at which the current density in the uniform part is found to be $j_{u}=0.3209$, which is very close to the corresponding value for $l_{d}=2 \pi$ (thus consistent with the weak-defect assumption), 


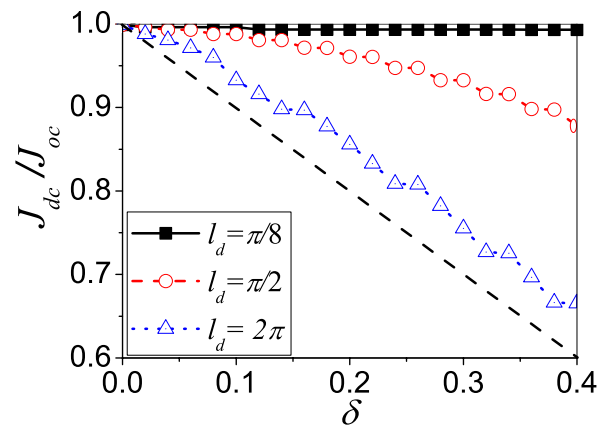

FIG. 7. (Color online) The normalized critical current $J_{d c} / J_{o c}$ as a function of defect depth $\delta$ for different values of defect length $l_{d}$, with system length $l=128 \pi$. The dashed line denotes $J_{d c} / J_{o c}=1-\delta$. The roughness of the curves arises from the discrete nature of the winding numbers and the currents for a finite system length.

but the current density at the narrowest point of the defect is $j_{\max }=j_{u} /\left(1-\delta_{c}\right)=0.6054$, which is well above $j_{o c}$. Such a large current density found at the boundary of metastability arises from the spatial correlation of the order parameter: a short segment of large current density is connected to and stabilized by the uniform segment of small current density. For an even smaller $l_{d}=\pi / 8$, the current-carrying state with $n=24$ will never lose its metastability because of the spatial correlation of the order parameter and consequently the effective "invisibility" of the defect.

To summarize, in the weak-current regime $\left(J_{d} / J_{o c} \rightarrow 0\right)$, $\Delta F_{d} / \Delta F_{o}$ decreases with increasing $\delta$ and the slope of such decrease increases with increasing defect length $l_{d}$. The largest slope of decrease is close to 1 because of $\Delta F_{d} / \Delta F_{o} \cong 1$ $-\delta$ for long defects, already shown in Fig. 5(a). Beyond this regime, our calculations show that for long defects, the decrease of $\Delta F_{d} / \Delta F_{o}$ with increasing $\delta$ is faster than $1-\delta$, i.e., $\Delta F_{d} / \Delta F_{o}<1-\delta$, as shown in Figs. 5(b) and 6. In particular, the stronger the current is, the faster the decrease of $\Delta F_{d} / \Delta F_{o}$ is, and hence earlier is the metastability lost at a smaller value of critical depth $\delta_{c}$.

Finally, we turn to the variation of critical current with defect strength. Figures 5 and 6 show the free-energy barrier $\Delta F_{d}$ to decrease with increasing defect strength. That is, the metastability of a current-carrying state would be weakened by increasing $l_{d}$ and/or $\delta$, and consequently the critical current would be reduced. In experimental studies, the critical current is one of the most important measurable quantities, and hence it is of interest to quantitatively investigate its variation with defect strength. For 1D wires of nonuniform CSA, there is no analytical expression for the critical current. Here, we present some numerical results obtained for defects of cosine profile. In Fig. 7, the normalized critical current $J_{d c} / J_{o c}$ (with $J_{o c}=\sqrt{4 / 27}$ as the critical current for the uniform wire of $\sigma_{o}=\sigma_{u}$ ) is plotted as a function of defect depth $\delta$ for different values of defect length $l_{d}$. It is readily seen that the critical current is reduced by increasing $l_{d}$ and/or $\delta$. It is interesting to note that for a given defect depth $\delta$, the strongest suppression of $J_{d c}$ is realized by relatively long defects $\left(l \gg l_{d} \gg 1\right)$ in the form of $J_{d c} \cong J_{o c}(1-\delta)$. This relation has been reasonably used in experimental studies. ${ }^{7}$ Physically, it is easy to understand this limiting behavior. If the defect is sufficiently long, then the narrowest section of CSA $\sigma_{u}(1-\delta)$ is no longer stabilized by the distant uniform parts with larger CSA and smaller current density. As a consequence, this weakest point would lose its (local) metastability when the current density there exceeds $j_{o c}=\sqrt{4 / 27}$, which corresponds to the critical current $J_{d c}=j_{o c} \sigma_{u}(1-\delta)$ $=J_{o c}(1-\delta)$. Shortening the defect would bring it to the stabilizing protection by the uniform neighbors and thus increase the critical current $J_{d c}$.

We would like to point out that the above results concerning the defect-related free-energy barrier and critical current are obtained by treating the dimensionless defect length $l_{d}$ as a control parameter, which is the real defect length measured and/or scaled by the temperature-dependent correlation length $\xi(T) \propto \varepsilon^{-1 / 2}$. It follows that, for a defect contained in a superconducting wire, its dimensionless length is actually temperature-dependent: $l_{d} \propto \varepsilon^{1 / 2}$. Consequently, a part of the temperature dependence of free-energy barrier and critical current comes from their dependence on $l_{d}$. The other part of the temperature dependence comes from the temperaturedependent energy parameter $g(\varepsilon) \propto \varepsilon^{3 / 2}$ and current unit $\sigma_{o} \xi^{-1} \alpha / \beta \propto \varepsilon^{3 / 2}$, obtained in dedimensionalizing the GL theory. Because $\Delta F_{d} / \Delta F_{o} \rightarrow 1$ in the limit of $l_{d} \rightarrow 0$ as $\varepsilon \rightarrow 0$, the defect-induced transition rate $\Gamma_{d}$ becomes negligible compared to that of the long, uniform part $\Gamma_{o}$ when the temperature is below but close to $T_{c}$. As the temperature is further lowered, $l_{d}$ and $g(\varepsilon)$ both increase. An increasing $l_{d}$ reduces the dimensionless free-energy barrier toward its lowest saturation value, and an increasing $g(\varepsilon)$ quickly raises the ratio $\Gamma_{d} / \Gamma_{o} \sim e^{g(\varepsilon)\left(\Delta F_{o}-\Delta F_{d}\right)}$. Together, they may lead to the dominance of $\Gamma_{d}$ in the total rate $\Gamma_{t}=\Gamma_{d}+\Gamma_{o}$, as shown in Fig. 4. The dependence of the dimensionless critical current on $l_{d}$ indicates that the temperature dependence of the critical current would deviate from the $\varepsilon^{3 / 2}$ behavior derived from the temperature dependence of the current unit in dedimensionalizing the GL theory. This $\varepsilon^{3 / 2}$ behavior of the critical current is usually used in determining the critical temperature $T_{c}{ }^{4,20,21}$

\section{CONCLUSION}

The string method has been used to study the thermally activated phase slips in nonuniform 1D superconducting wires, modeled by introducing a single geometric defect into an otherwise uniform cross section. The defect-induced phase slips are numerically computed and compared to those occurring in the (long) uniform part. The effects of a defect on metastable states, saddle points, free-energy barriers, prefactors, and transition rates have been quantitatively evaluated. In particular, it has been demonstrated that, in the experimentally relevant temperature range, the defect-induced phase slips with reduced free-energy barriers can dominate in the total transition rate. The variation of the free-energy barrier with defect length and depth has been investigated. Our numerical results show that, for weak currents, the freeenergy barrier associated with a relatively long defect 
$\left(1 \ll l_{d} \ll l\right)$ can be well approximated by $\Delta F_{d} / \Delta F_{o}=1-\delta$, but for strong currents (comparable to the critical current), the reduction of the free-energy barrier can be much faster than that of the cross-sectional area, i.e., $\Delta F_{d} / \Delta F_{o}<1-\delta$.

Experimentally, the relative inhomogeneity $\Delta \sigma / \sigma \sim \delta$ in cross section is expected to increase with ever decreasing (average) CSA of the nonuniform 1D wires. This implies a more important role played by the defects in ultrathin wires. It is our hope that the numerical results presented here are found to be relevant and useful by future experimental studies.

\section{ACKNOWLEDGMENTS}

This work was partially supported by the Hong Kong RGC under Grants No. HKUST 602904 and No. HKUST 602805 .
*Corresponding author. maqian@ust.hk

${ }^{1}$ W. A. Little, Phys. Rev. 156, 396 (1967).

${ }^{2}$ J. S. Langer and V. Ambegaokar, Phys. Rev. 164, 498 (1967).

${ }^{3}$ D. McCumber and B. Halperin, Phys. Rev. B 1, 1054 (1970).

${ }^{4}$ J. E. Lukens, R. J. Warburton, and W. W. Webb, Phys. Rev. Lett. 25, 1180 (1970).

${ }^{5}$ R. S. Newbower, M. R. Beasly, and M. Tinkham, Phys. Rev. B 5, 864 (1972).

${ }^{6}$ J. R. Miller and J. M. Pierce, Phys. Rev. B 8, 4164 (1973).

${ }^{7}$ For a review, see W. J. Skocpol and M. Tinkham, Rep. Prog. Phys. 38, 1049 (1975).

${ }^{8}$ M. Tian, J. Wang, J. Kurtz, Y. Liu, M. Chan, T. Mayer, and T. Mallouk, Phys. Rev. B 71, 104521 (2005).

${ }^{9}$ M. Zgirski and K. Yu. Arutyunov, Phys. Rev. B 75, 172509 (2007).

${ }^{10}$ W. E, W. Ren, and E. Vanden-Eijnden, Phys. Rev. B 66, 052301 (2002).

${ }^{11}$ W. E, W. Ren, and E. Vanden-Eijnden, J. Appl. Phys. 93, 2275 (2003).
${ }^{12}$ W. E, W. Ren, and E. Vanden-Eijnden, J. Chem. Phys. 126, 164103 (2007).

${ }^{13}$ T. Qian, W. Ren, and P. Sheng, Phys. Rev. B 72, 014512 (2005).

${ }^{14}$ H. A. Kramers, Physica (Amsterdam) 7, 284 (1940).

${ }^{15}$ R. Landauer and J. A. Swanson, Phys. Rev. 121, 1668 (1961).

${ }^{16}$ J. S. Langer, Phys. Rev. Lett. 21, 973 (1968).

${ }^{17}$ G. Henkelman, B. P. Uberuaga, and H. Jonsson, J. Chem. Phys. 113, 9901 (2000).

${ }^{18}$ M. B. Tarlie, E. Shimshoni, and P. M. Goldbart, Phys. Rev. B 49, 494 (1994).

${ }^{19}$ M. Tinkham, Introduction to Superconductivity (McGraw-Hill, New York, 1995).

${ }^{20}$ W. W. Webb and R. J. Warburton, Phys. Rev. Lett. 20, 461 (1968).

${ }^{21} \mathrm{R}$. Tidecks, Current-Induced Nonequilibrium Phenomena in Quasi-One-Dimensional Superconductors (Springer, Berlin, 1990). 